\title{
Manga109 Dataset and Creation of Metadata
}

\author{
Azuma Fujimoto \\ The University of Tokyo \\ fujimoto@hal.t.u- \\ tokyo.ac.jp \\ Yusuke Matsui \\ National Institute of \\ Informatics \\ matsui@nii.ac.jp
}

\author{
Toru Ogawa \\ The University of Tokyo \\ t_ogawa@hal.t.u- \\ tokyo.ac.jp \\ Toshihiko Yamasaki \\ The University of Tokyo \\ yamasaki@hal.t.u- \\ tokyo.ac.jp
}

\author{
Kazuyoshi Yamamoto \\ The University of Tokyo \\ k-yamamoto@hal.t.u- \\ tokyo.ac.jp \\ Kiyoharu Aizawa \\ The University of Tokyo \\ aizawa@hal.t.u- \\ tokyo.ac.jp
}

\begin{abstract}
We have created Manga109, a dataset of a variety of 109 Japanese comic books publicly available for use for academic purposes. This dataset provides numerous comic images but lacks the annotations of elements in the comics that are necessary for use by machine learning algorithms or evaluation of methods. In this paper, we present our ongoing project to build metadata for Manga109. We first define the metadata in terms of frames, texts and characters. We then present our web-based software for efficiently creating the ground truth for these images. In addition, we provide a guideline for the annotation with the intent of improving the quality of the metadata.
\end{abstract}

\section{Keywords}

manga, dataset, annotation tool

\section{INTRODUCTION}

With the increase in electronic publications of comics, there is a growing demand for processing of comic images. Potential applications of comic image processing include character recognition, multimodal search for images and text, and semantic understanding of story lines.

To develop and evaluate manga image processing techniques, it is important to have a publicly available dataset with detailed annotations. However, it is difficult to collect and use comic images for research purposes because comics are works of art and protected by copyright. To address this need, our group built the Manga109 dataset [2], [6], which is publicly available for academic research purposes, containing 109 comic books with permission granted by their creators. Manga109 provides many high-quality comic images, but it lacks detailed annotations such as texts, character names with regions of interest (ROIs), and frames. Such metadata can be a rich source of information by which we can train

In work is licensed under a Creative Commons Attribution International 4.0 License.

MANPU '16 December 04-04, 2016, Cancun, Mexico

(C) 2016 Copyright held by the owner/author(s).

ACM ISBN 978-1-4503-4784-6/16/12.

DOI: http://dx.doi.org/10.1145/3011549.3011551 machine learning algorithms and evaluate methods.

In this paper, we propose an annotation tool for comic images. With this software, users can crop rectangular areas and assign labels easily. The software is web-based, so it is suitable for large-scale annotation. In addition, the tool helps users annotate texts by using automatic recognition. The purpose of this project is to annotate metadata for all of the pages in Manga109.

\section{RELATED RESEARCH}

In the computer vision community, it is widely known that well-crafted image datasets with detailed annotations have played critical roles in improving image processing techniques; an example is MSCOCO [5]. For comic image processing, there have been several attempts to create a dataset with annotations, as follows.

Mihara et al. [7] proposed a way to annotate metadata for comic images. This work introduced a structure for metadata using a model for comics; annotation software for establishing metadata was also presented. However, the tool is a desktop application. Thus, it is not suitable for a largescale annotation project because users first need to install the software, which prevents many users from contributing to the project. We have addressed this barrier by making our software web-based for efficient annotation, so a large number of participants can join in.

eBDtheque [4] is a comic image dataset that includes metadata for panels, balloons, and text lines. eBDtheque is the first comic dataset with detailed metadata, but it contains a relatively small number of pages (100). Our goal is to create a large-scale dataset by annotating all of the pages in Manga109 (21,142 pages).

\section{MANGA109 DATASET}

In this section, we briefly explain the Manga109 dataset,

Table 1: Number of comic volumes in the decades or the genres in Manga 109
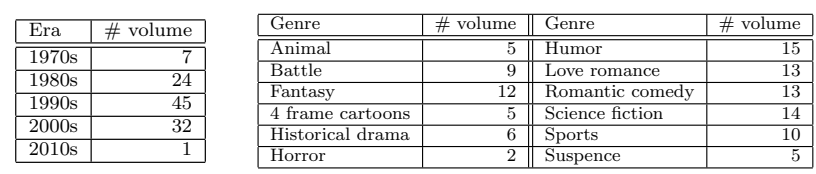


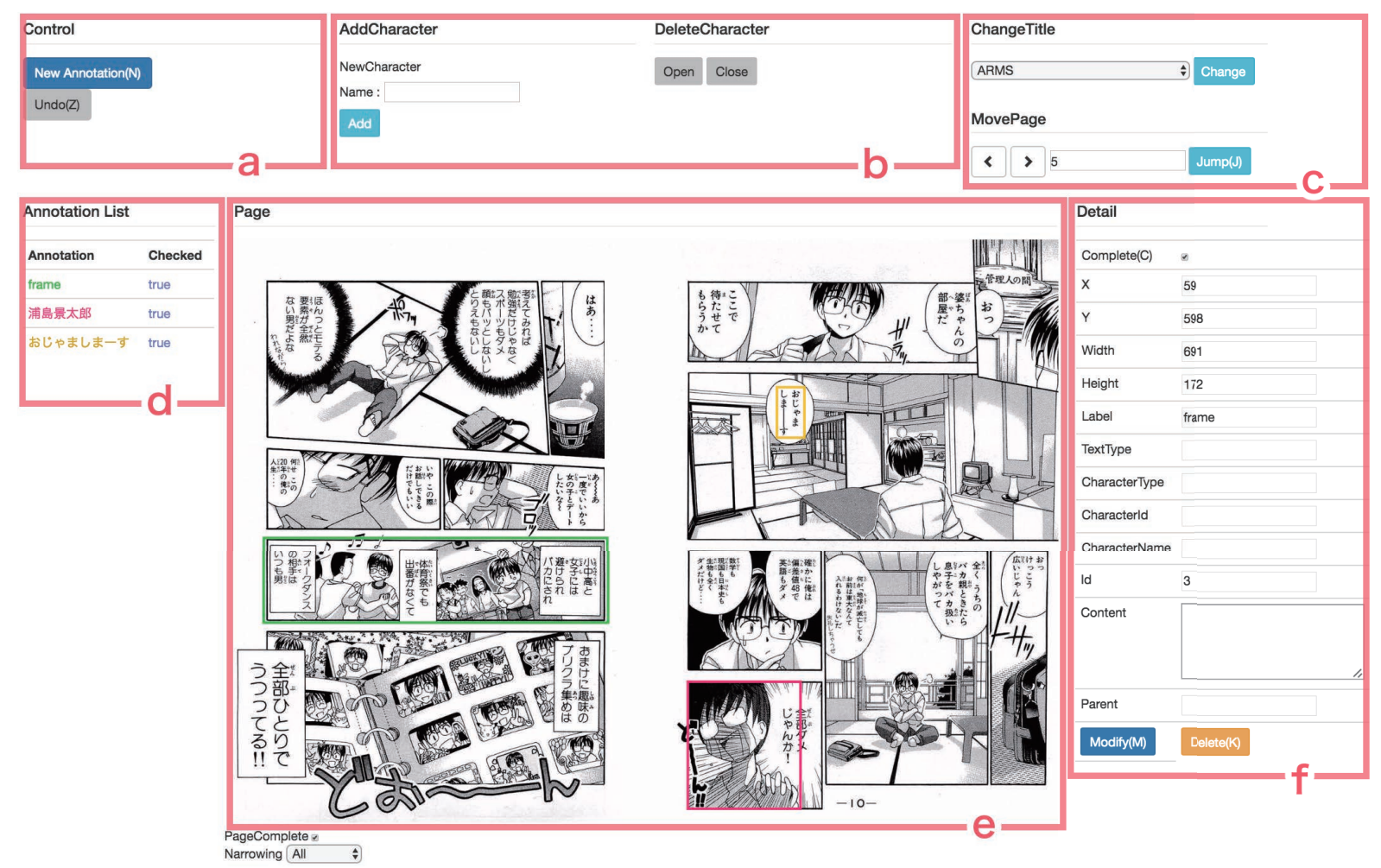

Figure 1: Software window areas: (a) New annotation and undo, (b) Character addition and deletion, (c) Title or page move, (d) Annotation list, (e) Page, (f) Detail

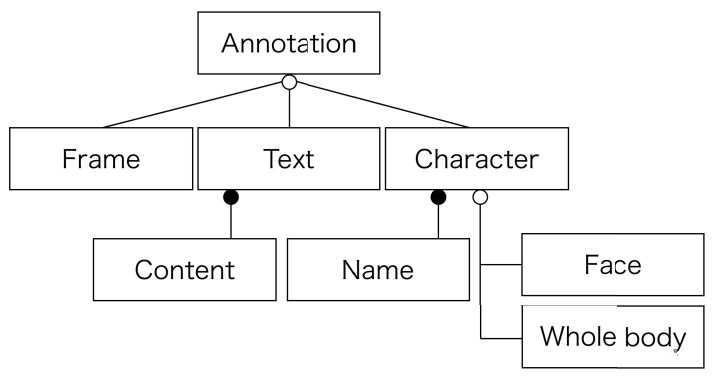

Figure 2: Annotation labels. A line with a white circle means that the parent is divided into categories (the children). A line with a black circle means that the child represents a property of the parent.

which is the dataset targeted for annotation. We created the Manga109 dataset, containing 109 Japanese comic books, for a total of 21,142 pages. Each comic book contains 194 pages on average. The comics were created by 94 professional creators. The dataset covers the era from 1970 to 2010 and includes various genres (e.g., science fiction, humor, military, and love and romance). Numbers of comics in the decades or the genres are shown in Table 1. Because permissions have already been obtained from the creators, the images can be freely used for academic research purposes with a notice of "(C) AUTHOR_NAME".

We established Manga109 with the help of Manga Library Z [1], managed by Ken Akamatsu. Manga Library Z is an organization that collects out-of-print manga; its collection

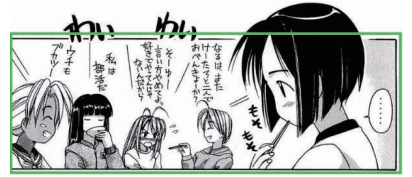

(a) Frame that contains a character that is placed partly outside the frame

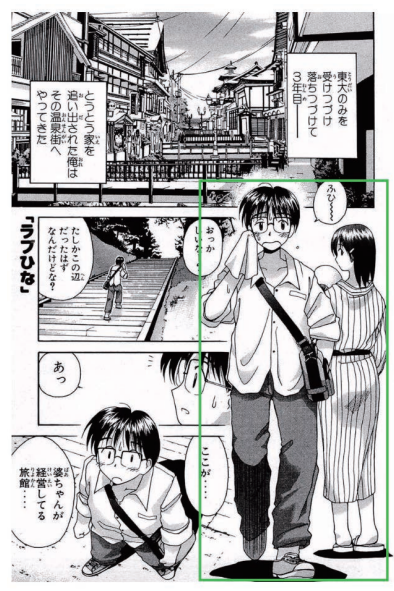

(b) Frame that has no bounding box

Figure 3: Protocol for designation of frames

contains over 4000 comics. From these comics, we selected 109 titles to construct Manga109. In selecting the comics, we used the following criteria:

- The comics should cover a wide range of genres and publication years.

- If a title had several volumes, we selected one volume from it. However, in cases where a title had more 


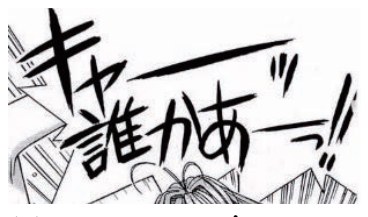

(a) A character's speech

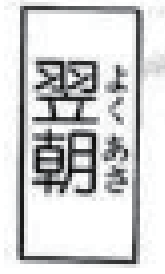

(b) Text explaining the time

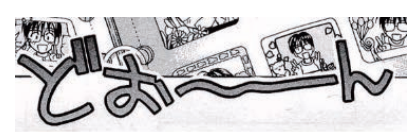

(a) Onomatopoeia

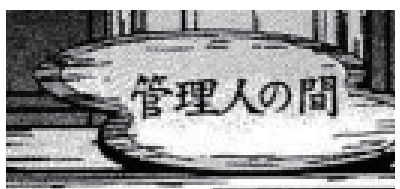

(b) Text drawn in the background
Figure 5: Excluded texts

than ten volumes, we selected the first and the last volume because the drawing style of a creator often changes after several volumes are published. In this way, the dataset may include images of different styles by the same creator, which could provide information of interest to researchers.

- The comics should not contain expressions that are irrelevant for the purposes of publishing academic papers, such as violent or sexual scenes.

\section{ANNOTATION TOOL}

In Fig. 1, we present a screenshot of our software for the efficient annotation of metadata in Manga109. In this section, we describe the structure of the metadata and the design of our software.

\subsection{Structure of the metadata}

Here, we define the structure of the metadata for a manga image. We define an annotation as a rectangular area with a label identifying a fundamental element of comics. We define three types of labels: "Frame," "Character," and "Text." Additionally, the "Character" label is further divided into "Whole body" and "Face"; we adopt this fine categorization of the character label because a face is the most important element for character recognition and detection.

Fig. 2 shows the label structure. An annotation with a text label contains "Content," such as the textual information in speech balloons. An annotation with a character label contains "Name," which is the name of the character.

\subsection{Guideline for the annotation}

In order to maintain the quality of the ground truth, a fixed guideline for annotation is needed. We have established a guideline for the annotation of frames, texts, and characters containing faces and whole bodies.
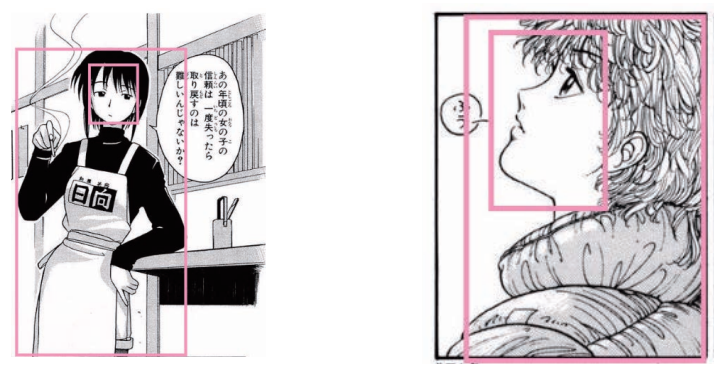

Figure 6: Included characters: whole body (entire part visible within the frame) and face of the character, annotated separately

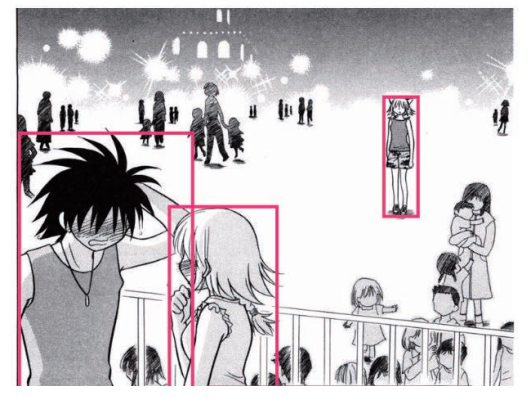

Figure 7: Excluded characters: Crowd characters without rectangles are not included.

- Frames: According to eBDtheque [4], frames "are defined as an image area picturing a single scene." We adopt this definition. An example of a frame is shown as a green rectangle in Fig. 1.

Fig. 3 shows cases in which a frame cannot be uniquely defined. In Fig. 3(a), part of the head of a character is placed outside the frame. In such a case, we simply designate the rectangular area for the frame as indicated by the green line. Fig. 3(b) shows an example in which a bounding box does not exist. In such a case, we create a virtual bounding box and use that as the frame.

- Texts: A text is defined as a group of written characters, such as text in a speech balloon, stage directions, or narration. We excluded texts that did not perform an important role in the story line of the comic. For example, we excluded onomatopoeia and text drawn in the background. Fig. 4 and Fig. 5 show instances of included texts and excluded texts, respectively.

- Characters: Character annotations are divided into faces and whole bodies. "Whole body" means the entire part of the character that is visible within the frame and is annotated for all characters, and "Face" is annotated for those characters whose face is clearly displayed in the page. Extras who do not perform an important role in the story line are not annotated. Fig. 6 shows the annotation of characters who have a visible face, and in Fig. 7, people in a background are excluded. 


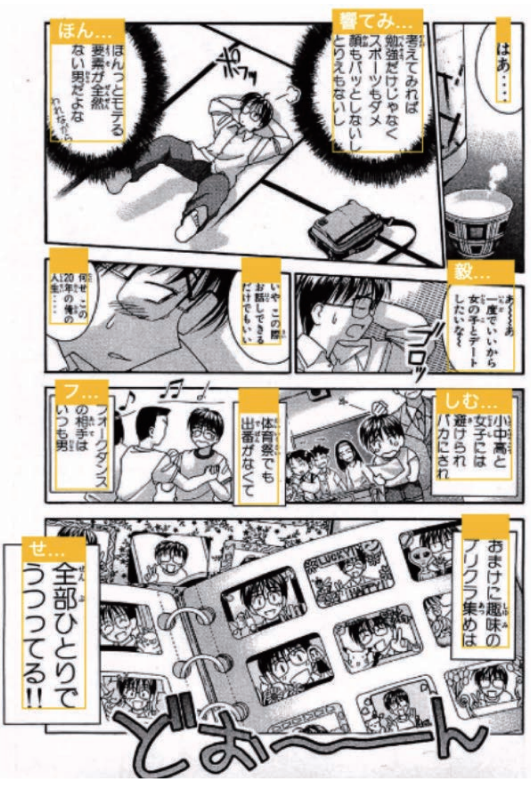

(a) Input image and the correct bounding boxes of texts
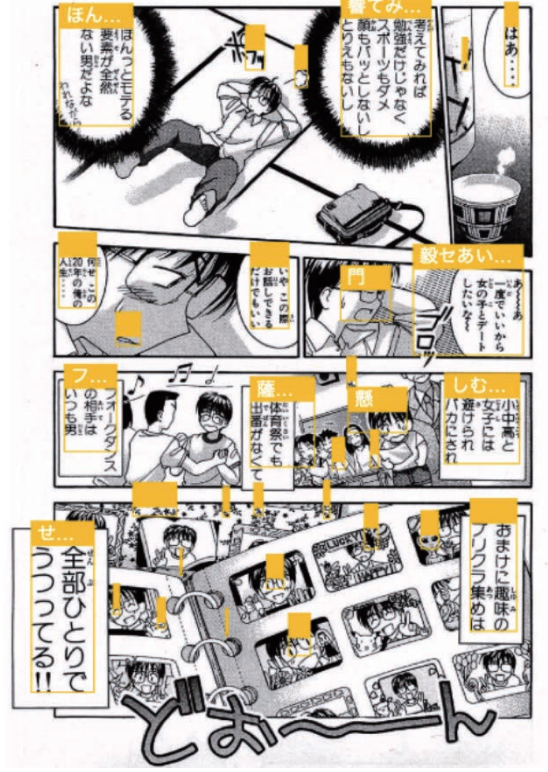

(b) Result of existing text recognition method
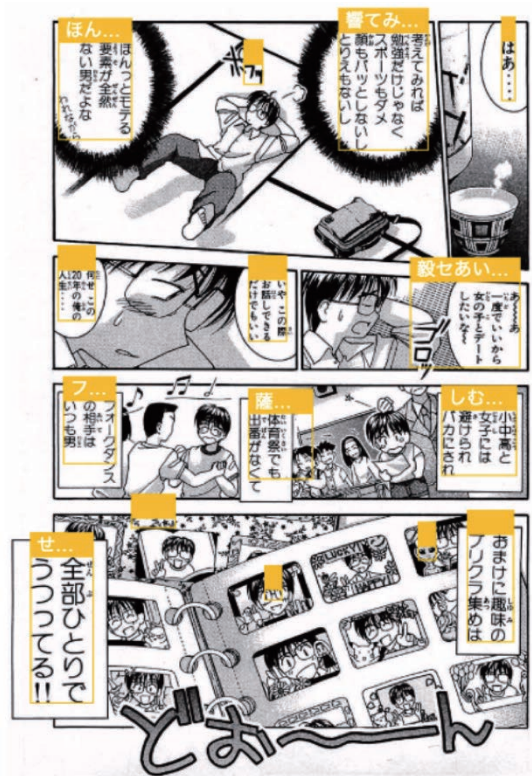

(c) Result after applying our filter

Figure 8: Result of filtering

\subsection{Summary of software design}

In general, the annotation of images requires a large amount of effort and many workers. For this reason, we developed a web-based program. The software is written in HTML and JavaScript, so it can be easily run on various devices without the need to install anything new.

This software is designed to easily allow the designation of a rectangular area and the assigning of a label. The software window is divided into six areas, as shown in Fig. 1. Users can view a page in the page area (e), and they can view all annotations for the current page in the page area (e) or in the annotation list area (d). When the annotations increase in number, the page area (e) often becomes confusing, and the annotation list area (d) is useful in these cases. Users can add a new annotation by pressing the New Annotation button in the new annotation and undo area (a), crop a rectangular area in the page area (e), and assign a label or provide more information in the detail area (f). When users edit existing annotations, they specify the target in the annotation list area (d) or the page area (e), edit a bounding box in the page area (e), and edit the information in the detail area (f). Everything that users may wish to do can be executed in one window, with no screen transitions or scrolling needed. Users can also use keyboard shortcuts as an alternative to the mouse.

\subsection{Automatic recognition of texts}

In the process of annotation, the typing of texts is the most time-consuming process for annotators. To help speed the typing process, we apply a text recognition method [3] to input images in advance. The output of the text recognition algorithm (a text with an ROI) is automatically recorded in the system for the annotators' use. However, if the text output from the automatic recognition algorithm is not correct, the annotators can revise these annotations or simply discard them and create new annotations.

The quality of recognition is not always perfect. For example, several fragments of short texts or regions may be produced. Therefore, we filter out such noise in advance: If the size of the text area is less than 200 px or the number of letters is less than three, we delete this text area from the results. Fig. 8 shows that the result of the automatic recognition algorithm is greatly improved by our filter. Using the above method, annotators are only needed for revising errors in the results, and consequently the efficiency of the work will improve.

\section{EXPERIMENT}

To investigate the effectiveness of our software, we conducted user studies. Three users annotated metadata for comic images using our software, and we measured their work time. The setting and procedure for the experiment were as follows.

1. The targets in the experiment were three comics, "LoveHina," "ARMS," and "Arisa2," shown in Fig. ??. All of these comics are contained in Manga109.

2. First, we asked participants to use the software for five minutes to practice its use.

3. Participants annotated metadata for frames, texts, and characters. In the annotation of texts, they used the results of the automatic recognition algorithm. When the name of a character was not written in the page, they searched for it in neighboring pages. If they could not find the name, they assigned a general word, e.g., "other" or "man."

4. The amounts of time required to finish were measured. 


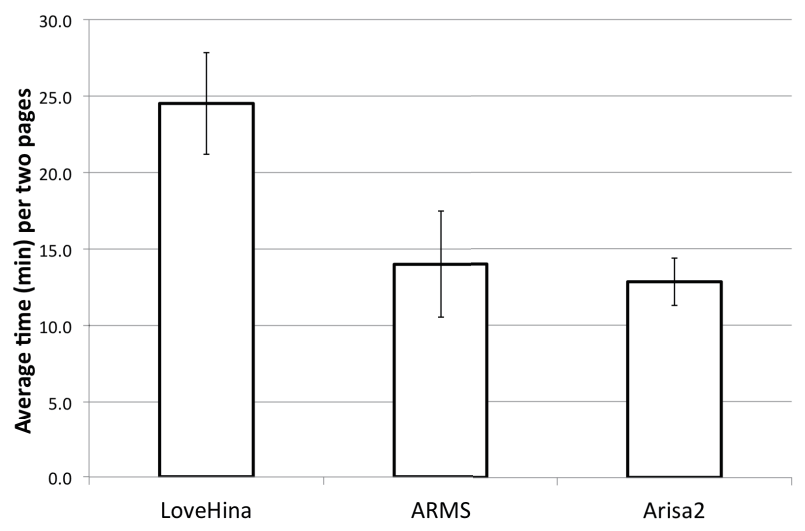

Figure 9: Time required for annotation

The result of the experiment is shown in Fig. 9.

Fig. 9 shows that the average of the time required for the annotation of two pages was 17 minutes. A typical comic book has about 200 pages, so one comic book would require about 28 hours to annotate, and Manga109 would require about 3000 hours to annotate with this software.

On the other hand, Fig. 9 also shows that the times required varied a great deal from comic to comic. We presume that the times depended on the number of elements in the comic. For example, a page in "LoveHina" has many characters and texts, whereas a page in "Arisa2" has a smaller number of elements.

\section{CONCLUSION AND FUTURE WORK}

In this paper, we have presented the Manga109 dataset of comic images, containing numerous images. This dataset is publicly available for use for academic purposes. We have also presented our web-based software for efficient annotation; in addition, we have given the structure of the metadata and provided a guideline for annotation.

With this software, approx. 70 people contribute to the creation of annotation. The annotation of Manga109 will be built in near future.

\section{ACKNOWLEDGMENTS}

This work was supported by the Strategic Information and Communications R\&D Promotion Programme (SCOPE).

\section{REFERENCES}

[1] Manga library z. http://www.mangaz.com/.

[2] Manga109. http://www.manga109.org/.

[3] Y. Aramaki, Y. Matsui, T. Yamasaki, and K. Aizawa. Text detection in manga by combining connected-component-based and region-based classifications. IEEE ICIP, 2016.

[4] G. Clément, R. Christophe, M. Antoine, A.-B. Farid, B. Karell, B. Alain, B. Jean-Christophe, L. Georges, O. Jean-Marc, and R. Arnaud. ebdtheque: A representative database of comics. ICDAR, 2013.

[5] T.-Y. Lin, M. Maire, S. Belongie, J. Hays, P. Perona, D. Ramanan, P. Dollár, and C. L. Zitnick. Microsoft coco: Common objects in context. In European Conference on Computer Vision, pages 740-755. Springer, 2014.
[6] Y. Matsui, K. Ito, Y. Aramaki, T. Yamasaki, and K. Aizawa. Sketch-based manga retrieval using manga109 dataset. CoRR, abs/1510.04389, 2015.

[7] T. Mihara, M. Nagamori, and S. Sugimoto. A metadata model for representing story and visual structure of digital manga. IFAT, 2011. 